\title{
Making Humanoid Robots More Acceptable Based on the Study of Robot Characters in Animation
}

\author{
Fatemeh Maleki*, Zeinab Farhoudi** \\ * Departement of Animation, Tehra Art University, Tehran, Iran \\ ** Departement of Computer Engeniering, Science and Research Branch of Azad University, Tehran, Iran
}

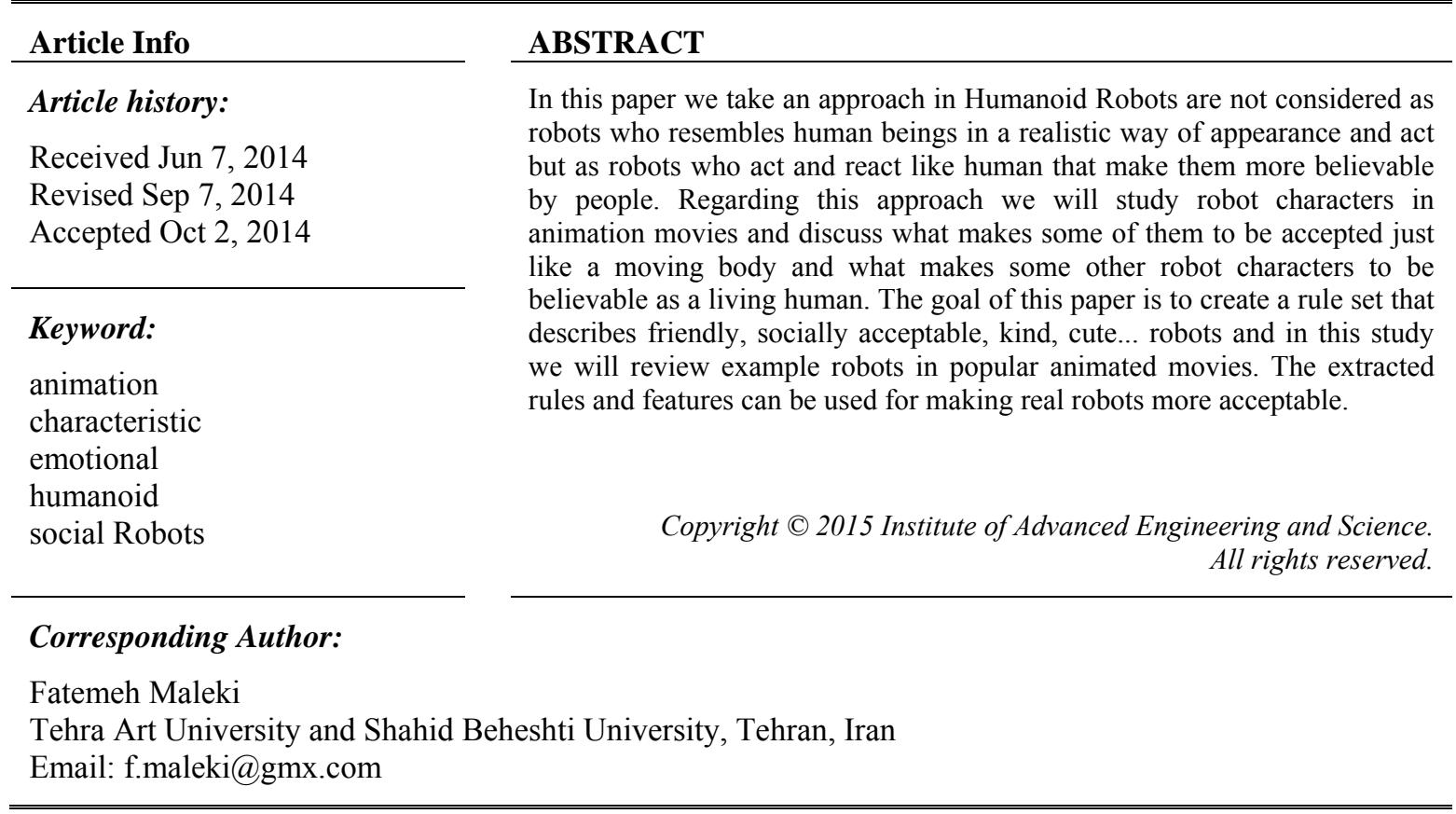

\section{INTRODUCTION}

Walt Disney says, "When people laugh at Mickey Mouse, it's because he's so human; and that is the secret of his popularity." Although the characters in any animation could be objects, toys, plants, or animals, but most of the time, regarding the story, they are not themselves but considered as a humans to audience's conception. Mickey Mouse is not a mouse but a human with tail, big ears and mouse-like face. Besides to these facts about identity of characters, a character has a face of object, animal, human, etc. If we focus on robot characters in animation movies sometimes they are considered as moving objects without any feelings but some other even including robots without humanoid faces and bodies, show emotions and react in a way that their audience believe them as a characters being familiar with for years [1-9].

In 2012, Disney lab developed a humanoid robot which has the capability of playing catch and juggling (Disney Research). Besides the scientific and engineering aspects of the robot such as artificial intelligence, face detection and mechanics, it has also interesting animations after failure and missed catches such as a shoulder shrug, shaking the head, and looking down [1]. This animation gives some characteristics to it (Figure 1). 


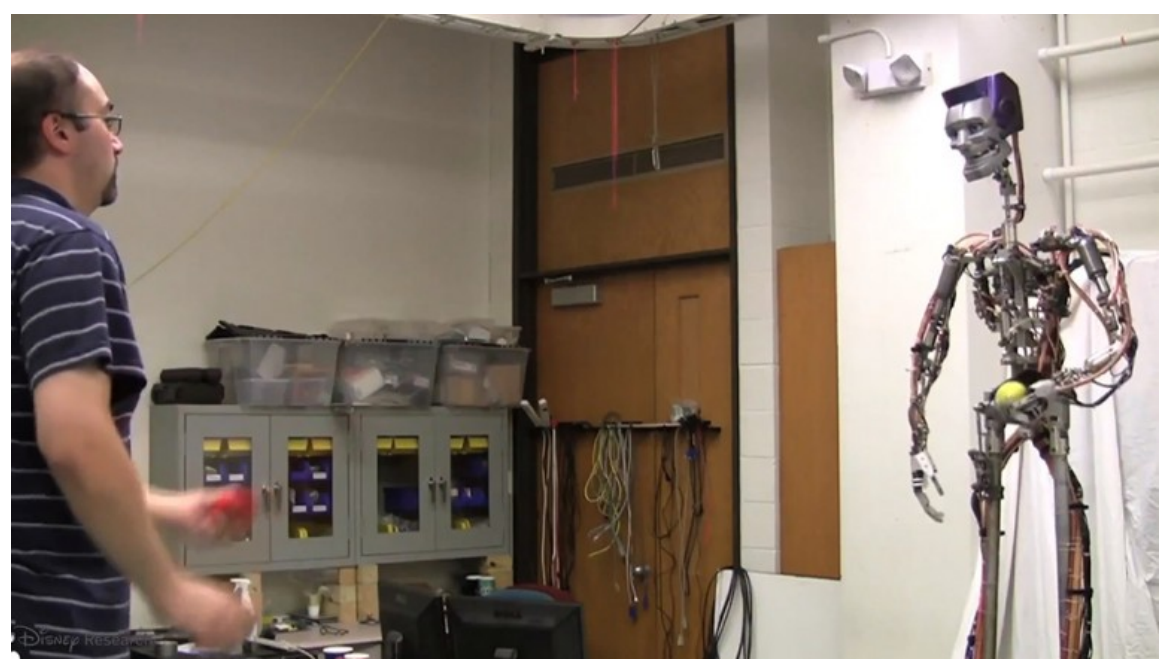

Figure 1: The juggling robot

Russel and Norvig utter four possible goals to pursue in artificial intelligence: 1) Systems that think like humans. 2) Systems that think rationally. 3) Systems that act like humans 4) Systems that act rationally [2]. To make a humanoid robot may not necessary mean to make a perfect and realistic robot: perfect in acts perfect in face, and perfect in emotional faces. Not being perfect makes it more possible to be different among others and that is what exists between human beings and forms the difference, characteristics and identity. Some features can be defined, and point is to decrease and increase some features in different robots to give them identity and make them more accepted by people. Although the shape and mechanics of motion is a matter but more is the acts and reactions.

Considering the animation Gagarin (1961) or the movie I, Robot (2004), all the caterpillars look alike and all the robots has the same appearance, but different acts and reactions of the main characters make them accepted as more look alike human. The how's of implementation of this differences can be discussed in technical topics, there could be many ways such as assigning a factor to some features or assigning factors to learning aspects that can be set for each robot specifically.

To find out what can help toward the goal of making believable robots and extract the rules for design,act and emotional reaction we will study famous robot characters in some long animation movies including: Robots (2005), WALL-E (2008), Megamind (2010), The Incredibles (2004), Pinocchio3000 (2004), Meet the Robinsons (2007), 9 (2009), Coraline (2009), Treasure planet (2002), Jimmy Neutron: Boy Genius (2001), Astroboy(2009), Iron Giant (1999), Castle in The Sky (1986).

This study can be useful in social and emotional robot study fields, and also can be used in helping children diagnosed with autism as " Children diagnosed with Autism Spectrum Disorders can find communication with others difficult. However, their relationship with machines is another story completely. Because of predictability and reduced external stimuli to process, technology often attracts children with autism. They find computers and other technological devices comforting, motivating, and engaging when it comes to areas like social interaction and communication." (Beyond.com) Dr Karen Guldberg, from the University of Birmingham's School of Education's Autism Centre for Education and Research hopes they're right. She was quoted in The Daily Mail as saying, "We have been looking at how technology can support pupils with autism to communicate more effectively." She adds, "Pupils and teachers are experimenting with the robots and other technologies in a developmental way and they are showing significant benefits for the classroom." (Beyond.com)

We will study robots in animation, some even may not be realistic but accepted as a friendly character, to export ways for making emotional and social robots. Considering all realistic characters animated with motion capture, sometimes they have gain less success due to suffering from a theory called The Uncanny Valley proposed in 1970 by Masahiro Mori [4]:

He envisioned people's reactions to robots that looked and acted almost human. In particular, he hypothesized that a person's response to a humanlike robot would abruptly shift from empathy to revulsion as it approached, but failed to attain, a lifelike appearance. This descent into eeriness is known as the uncanny valley. 

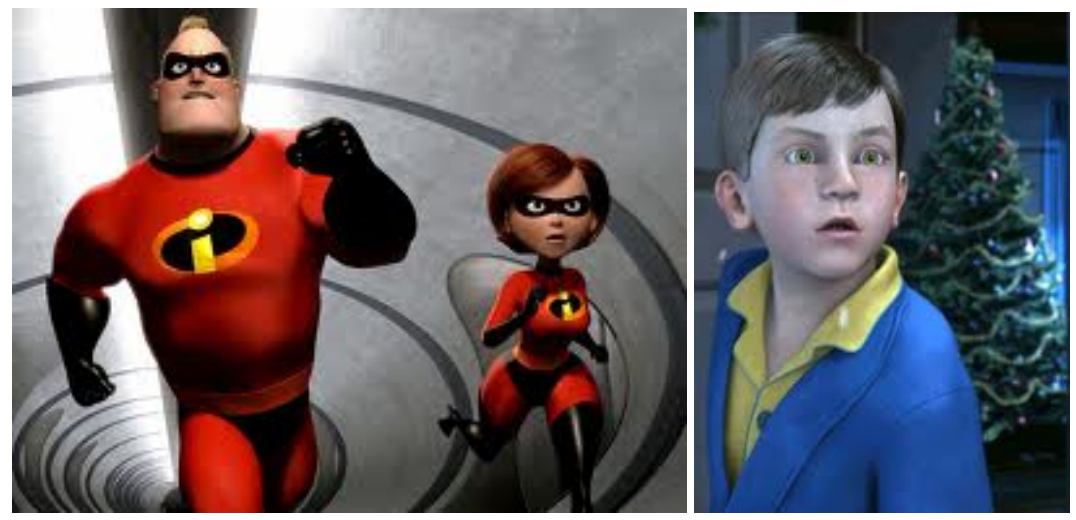

Figure 2. Incredible in contrast with The Polar Express, as it is seen the uncanny valley is more sensed in The Polar Express with more realistic characters than in Incredible.

\section{PATHIC FEEDBACK}

In this section we study the robot characters' acts and reaction and study it in specific parts of facial, body, sound and characteristics.

\subsection{FACIAL EXPRESSION}

This part studies the interactions and emotions shown on face of robot.

\subsubsection{EYES}

Eyes are of great importance in facial and emotional expression. This part talks about different shapes of eyes in characters although not realistic but with feelings and will discuss the aspects considered in eyes' or eyelids' and eyebrows' movement to give feeling to them.

\section{a) Ligh Eyes}

In WALL-E (2008) many of reactions are induced by Eva's light eyes. Eva has a simple light board, with eyes drawn on it.

The advantages of this would be:

- faster reaction when mechanical limitations exist.

- more flexible and asymmetric reaction can be implemented.

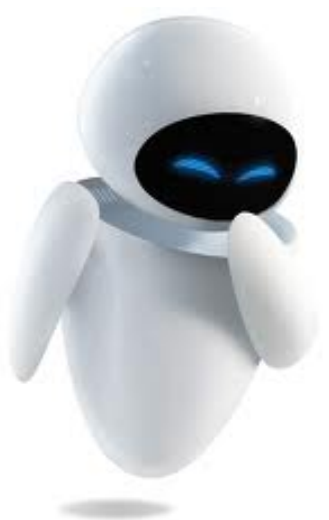

Figure 3. Eva (WALL.E, 2008)

When the size of robot increases and we get less involved in its facial emotions, just two circles or just two light points are used, for example in Astroboy (2009) there is a robot that has just two shining circles as its eyes and ZOG, a friendly robot with a large body and very small head has just two spheres as its eyes. 


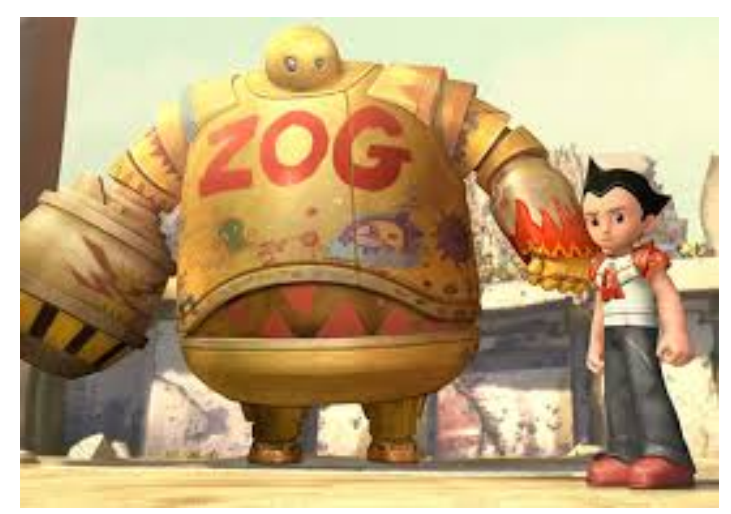

Figure 4. ZOG (Astroboy, 2009)

Another example of light eyes can be sweeper robots in WALL-E (2008), that light board shows the eyes' current form.

\section{b) Telescopic eyes}

Many robots in animations have two cylinders as their eyes. Iron Giant (1999), 9 (2009), Meet the Robinsons (2007), Treasure Planet (2002), Robots (2005) are examples of these animations. Using telescopic eyes give lower view angle to the visitors to diagnose the robots current eyes' form, so it can be a good trick to just concentrate on implementation of they appear in frontal view. The characters mentioned all has lower and upper lids or spiral lids to cover some parts of eyes and give emotions to them. Characters Eyes in 9 (2009) has dark circle inside as iris but in Iron Giant (1995) the robot has no iris but when it is needed to show the direction of look, two center lights in the eyes move to corners of the circles of eyes.

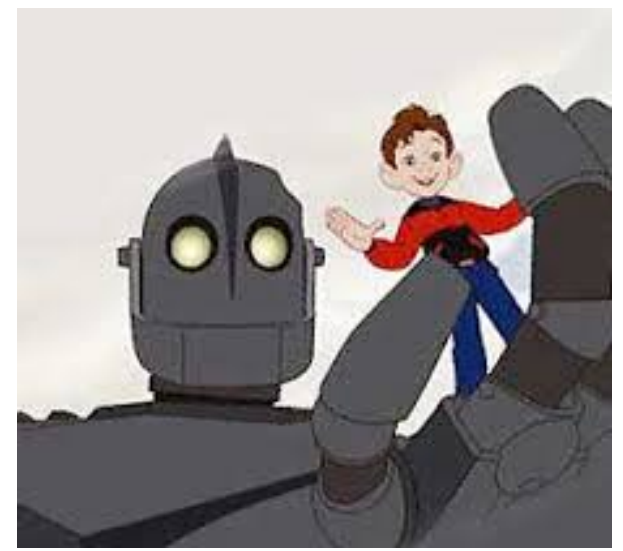

Figure 5. Iron Giant (Iron Giant ,1995)

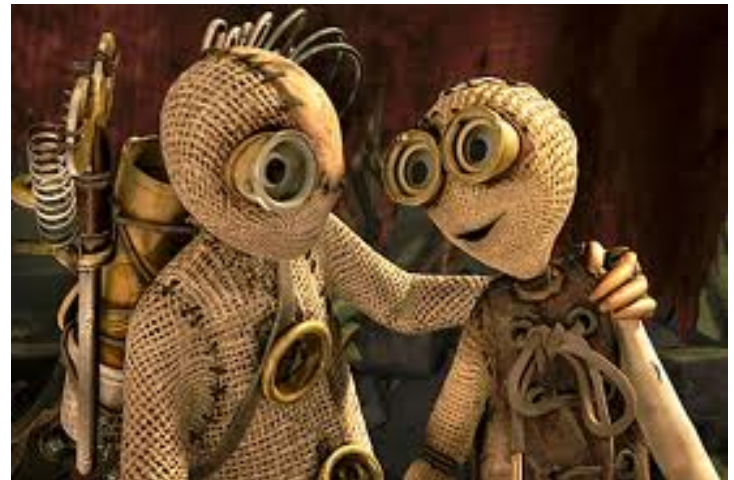

Figure 6. $9(9,2009)$

It remains unknown why we generate spontaneous eye blinks every few seconds, more often than necessary for ocular lubrication. Because eye blinks tend to occur at implicit breakpoints while viewing videos, we hypothesized that eye blinks are actively involved in the release of attention [5]. This is a great finding to be used in robots not just make them regularly blink or just blink when a change in direction of the look is applied on eyes.

\section{c) With eyeballs}

A cute example is the servant robot in Astroboy (2009) which has eyes with eyeballs. Pinocchio in Pinocchio3000 (2004) also has eyeballs. It is important to note these kind of balls are used in a not realistic look that is similar to the robot's caricatured faces. 


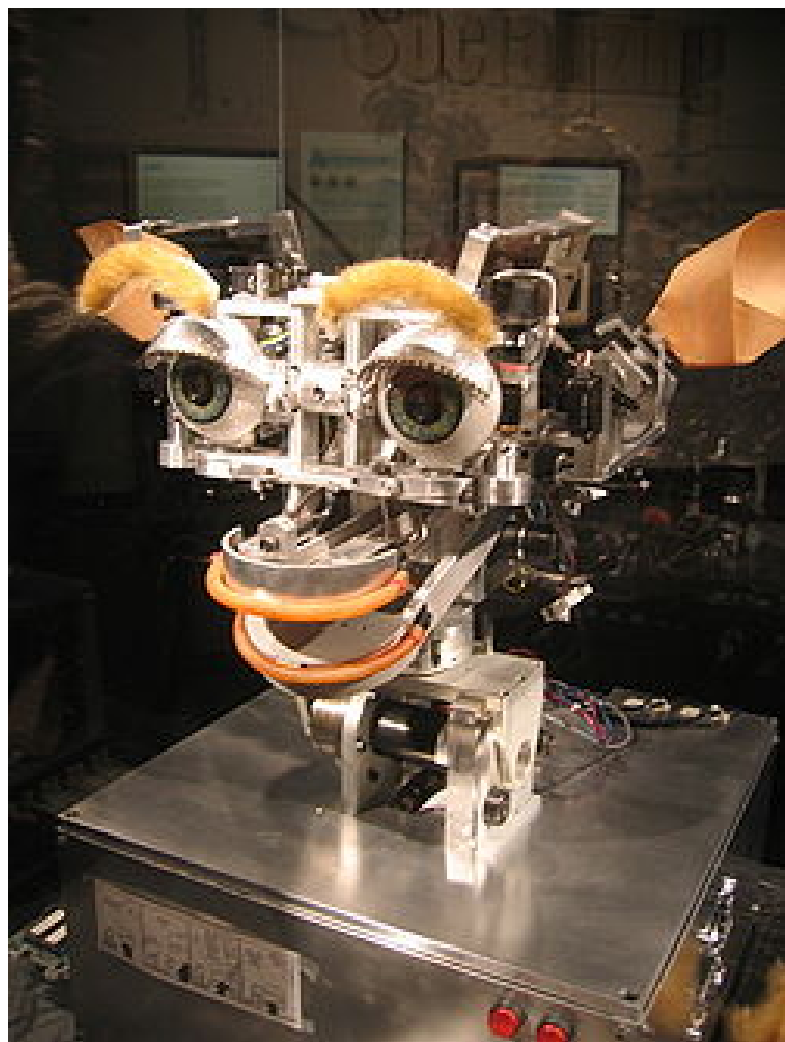

Figure 7: Kismet Robot (source: http://en.wikipedia.org/wiki/Kismet (robot))

\section{No eyes}

AUTO a character in WALL-E (2008) which is inspired by HAL in 2001: A Space Odyssey (1968) has just a central red light. A destructor robots in Megamind (2010) and 9 (2009) also have a central circle that attracts attention. In Iron Giant (1995) when the robot turns out from a good humanlike robot to just a defensive robot, the head is hidden and again a central circle is the main point of attention. The hat robot in Meet the Robinsons (2007) also has one LED semi-eye. These robots are all considered as machines and bad characters so it seems that placing a single light as the robots eyes should be avoided due to shared negative view of audience about it. In Monsters, Inc. (2001), a character named Mike also has one eye but the eye has emotions shown by eyelid and eyebrow and eyeball resembling real eye. In Coraline (2009), the Other Mother and Other Father has button eyes in normal size head but the emotions are shown by eyebrows and other facial expressions. 


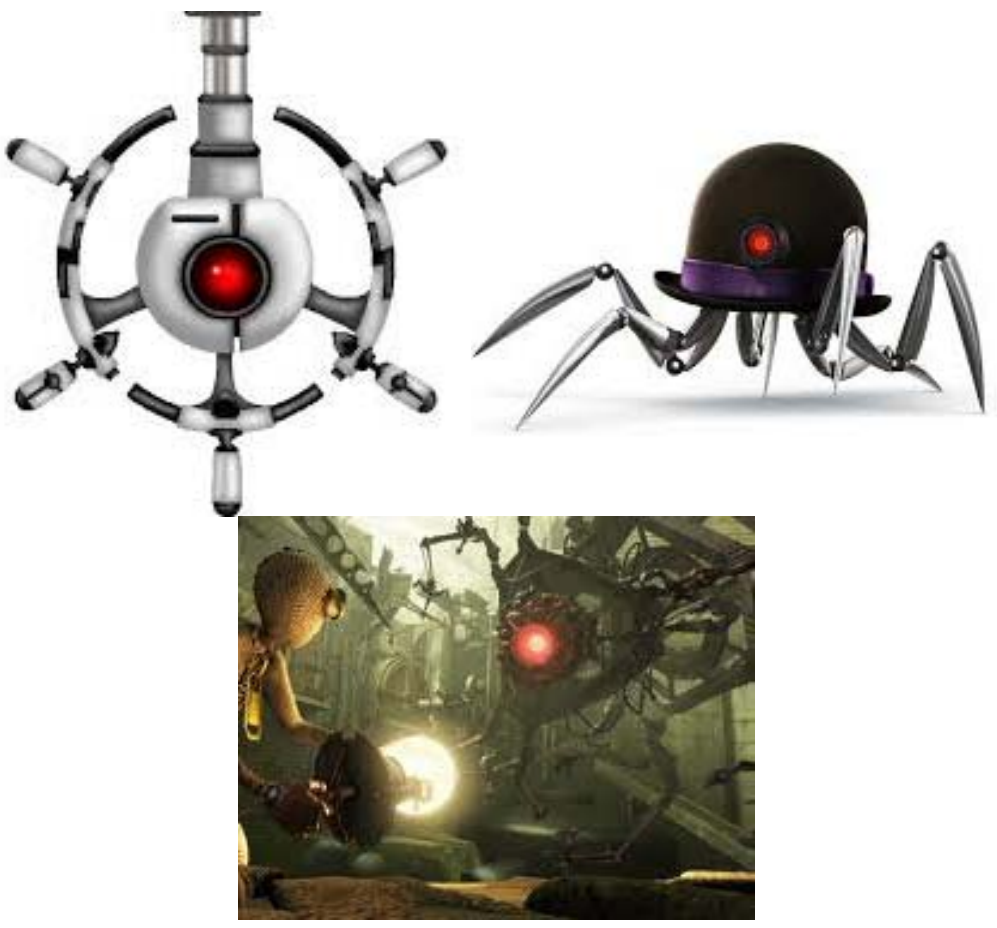

Figure 8: Auto (WALL.E, 2008) Figure 9: hat (Meet the Robinsons ,2007) Figure 10: robot (9, 2009)
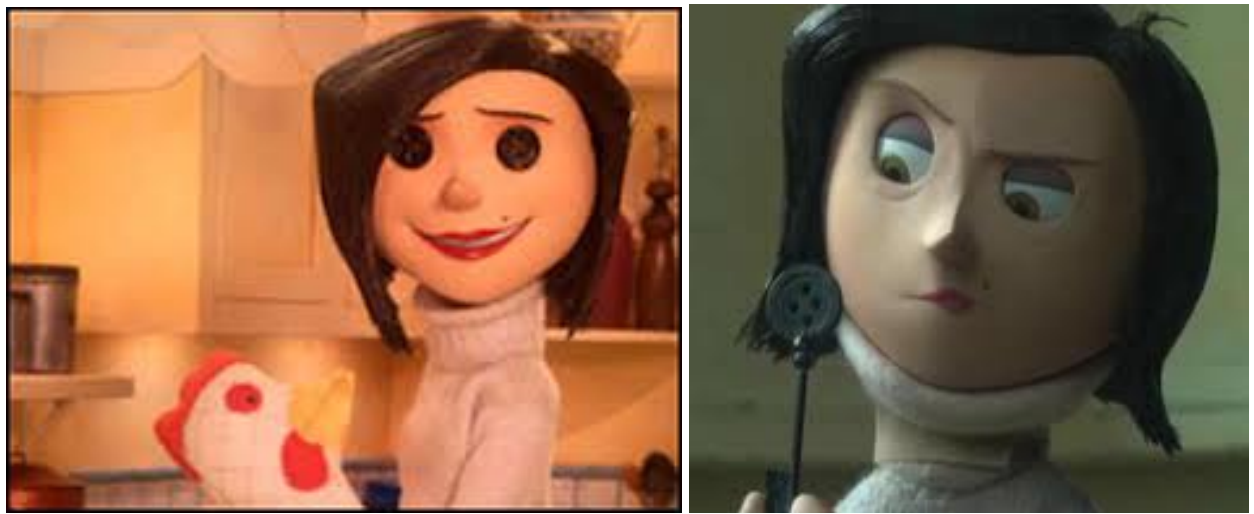

Figure 11: button Eye mother vs real mother (Coraline, 2009)

\subsubsection{MOUTH}

Lips has important role in making character believable. Sometimes we even look directly on the speaker's lips. Applying lip sync is very important. In cases where the mechanical speed of mouth has limitations display boards or even colored display boards can be used to show the lips form. For the second step the try could be on the whole body movement since in fact lip sync in not just about lips movements on voice but the whole body including face and hands.

Mouth are important in communicating children with autism too, as Dirk Neumann [6], et. al say:

People with autism are impaired in their social behavior, including their eye contact with others, but the processes that underlie this impairment remain elusive. We combined high-resolution eye tracking with computational modeling in a group of 10 high-functioning individuals with autism to address this issue. The group fixated the location of the mouth in facial expressions more than did matched controls, even when the mouth was not shown, even in faces that were inverted and most noticeably at latencies of 200-400 ms. Comparisons with a computational model of visual saliency argue that the abnormal bias for fixating the mouth in autism is not driven by an exaggerated 
sensitivity to the bottom-up saliency of the features, but rather by an abnormal top-down strategy for allocating visual attention.( Dirk Neumann, et. al, 2006)

The lips of extraterrestrial characters in Jimmy Neutron: Boy Genius (2001) and also the two robots who arrest Jim Hawkins in Treasure planet (2002), have display board as their mouth, but since they are not designed to be good characters the Display board just show sound waves.

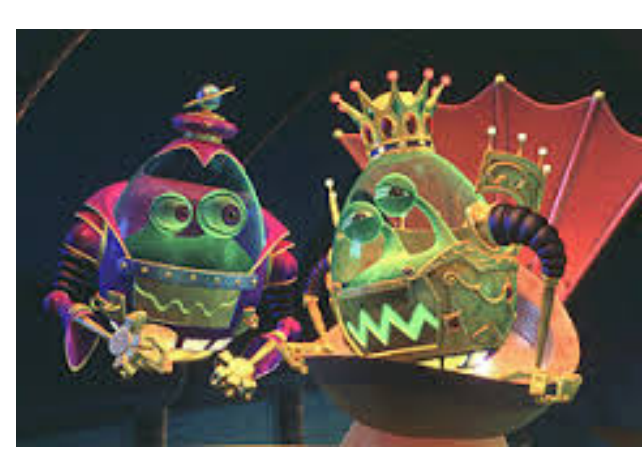

Figure 12: space creatures (Jimmy Neutron ,2001)

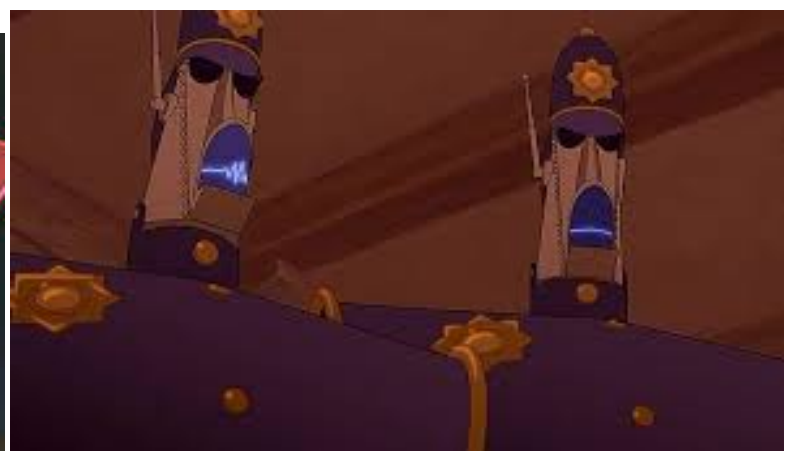

Figure 13: robots (Treasure planet, 2002)

Some robots may have a simple mechanical separate jaw as in Astroboy (2009).

\subsection{BODY LANGUAGE AND ROBOT'S EXPRESSION}

we considered facial part and facial expression in the previous section, another important thing that can define someone's characteristics is body language, they way one acts and use head, legs, hands for doing a work or showing emotions.

\subsubsection{DESIGN}

Some robots in animations such as Robots (2005), Meet the Robinsons (2007), Castle in the Sky (1986) or Omnidroid robot in The incredible (2004) have arms, legs and necks more like rubber hose animation with simple flowing curves and without articulations, but it may seem hard to be implemented that needs to control many small motors' rotation.

In Robots (2005) the spring character near gate has no legs, but the exaggerated springlike movement of his body gives him a great funny appealing characteristic, this model can be used where the making of legs and humanlike walking can be postponed or ignored but also want to make a robot with characteristics.

In Megamind (2010) and Jimmy Neutron: Boy Genius (2001) consequently a fish with a robot body and some extraterrestrial characters exists that are robots mixed of a life real beings that have robotic body. Not all the time the body parts should be integrated as Eva in WALL-E(2008), robot in Iron Giant (1995), Jimmy's dog in Jimmy Neutron: Boy Genius (2001), and a robot that can hold his head in hand in Robots (2005) can propose the idea of unconnected body parts. Unusual body proportion can also make weak and unusual actions be more easily accepted by visitors and also giving characteristics to the robot. Orrin in Astroboy (2009) has no legs and has a little hump which matches his character and also make it cute proposing different types of body design. 


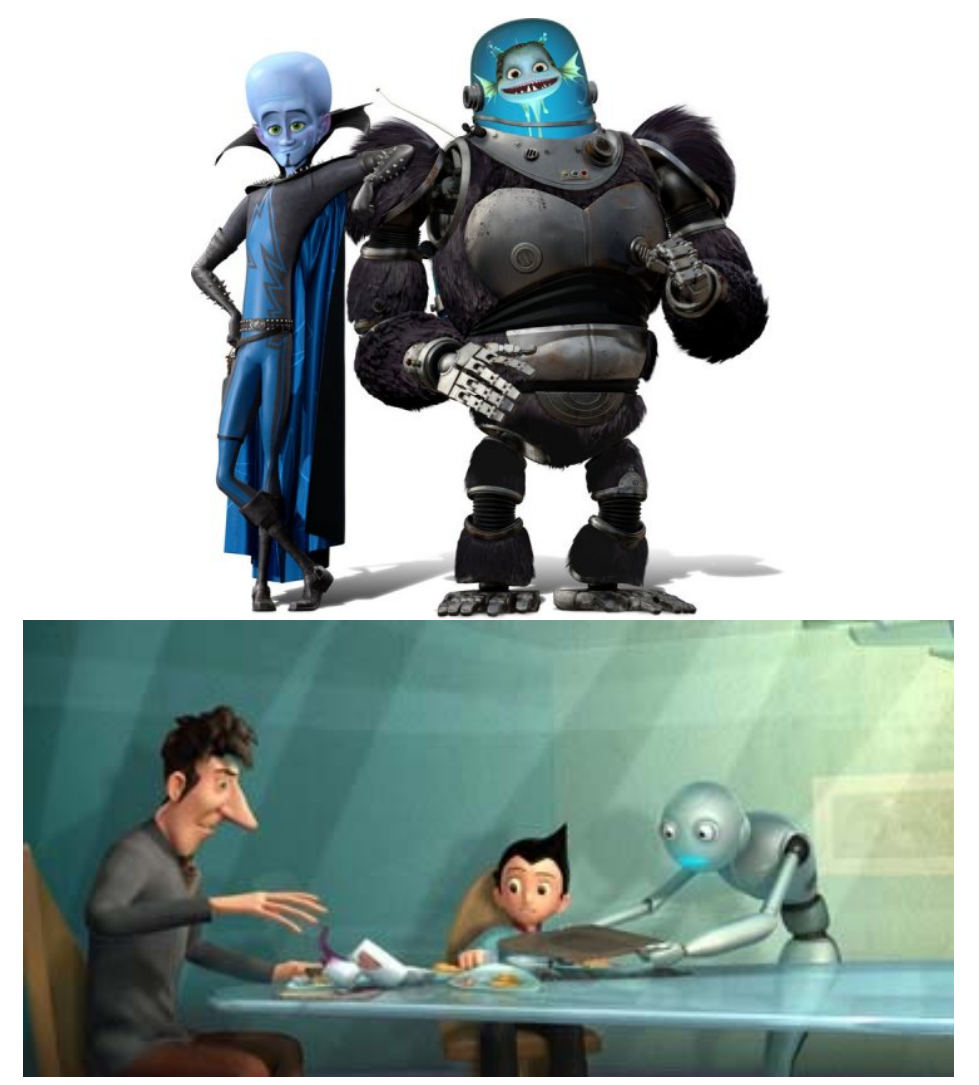

Figure 14: minion (Megamind ,2010)

Figure 15: orrin (Astroboy, 2009)

\subsubsection{ACTION}

Overlaying of actions is an important thing in human beings life, we close the dresser's door but before finishing it turn our head back ready to walk away from it.

People sometimes do some actions while doing another action that may not be necessary for a robot to do all actions together, for example we may frown while lifting a heavy thing or some may take their tongue tip out while concentrating on doing some precise work. Defining some unnecessary set of actions for doing some works can also help us give robots special characteristics, different sets for different robots. It is also related to Body Language studies, closing eyes while slow talking can show pleasure. In Megamind (2010) the robot bows near the sitting man while talking to him, this may appear unnecessary for a robot that can increases his volume, but this body language and set of actions can have psychological effect on the visitor. As another example of set of actions, in Robots (2005) two robots whisper, holding hand in front of mouth and look forward instead of looking at each other, meant to show the conversation is done hidden.

In 9 (2009), one character says, "follow me" and accompany it by his hand movement. Deaf-community sign language also can be studies and be a great source to be used. A robot that talks and also moves its hands according to the speech can have better effect.

In Iron Giant (1995), the robot put his hand under the chin. Setting a robot to do this or nodding while what the speaker says takes too long can have positive effect on the speaker.

\subsubsection{IMPORTANCE OF BETWEEN}

As like the speed of uttering a sentence can have different meanings, the speed and also change in speed of doing an action can also make it more natural. An example of importance of change of speed instead of actions with regular speed is in Jimmy Neutron: Boy Genius (2001) when the robot dog converts to just a loud speaker phone, his head start to rotate steadily clockwise and counter-clockwise like a radar antenna.

In WALL-E (2008), in a special shot that Eva is still a robot with no feelings, Eva starts laughing, although her laugh's sound, body movement and eyes shape are all so natural but we still feel her as a robot. The reason of this may be due to that the laugh follows another action suddenly without any action 
connecting them. Trying to show a robot more believable defining some actions between two opposite emotional actions can help to decrease this feeling.

Sometimes the robot may do nothing, in Iron Giant (1995), when the robot is free and bored he starts to open and close a car's door slowly and repeatedly. Defining some actions to be done while free and idle can make the robots appear more than just robots.

Using animation tweleve principals is another point as Breeman,"We proposed to apply principles of traditional animation to make the robot's behavior better understandable. Examples from our user-interface robot iCat were given to illustrate this idea." (van Breeman, 2004)

\subsubsection{ANIMATION PRINCIPLES OF ACTION}

Utilizing of animation principles can also help the robot to have a more acceptable movements, both considering the physics and exaggerations in it and also considering the psychology of body language that is the people are familiar with those exaggerated movements in body by watching many animations. Breeman studies applying of 12 animation principles such as Squash and stretch, Anticipation, Straight Ahead Action and Pose-to-Pose, Follow Through and Overlapping Action, Slow In and Slow Out, Arcs, Secondary Action, Timing, Exaggeration, Solid Drawing and Appeal on robots. (Breeman, 2004)

The acceptance of user-interface robots as "social friend" depends among others on the ability of the user to understand the robot's behavior - to understand what it is doing and thinking. Body gestures are a natural channel to communicate this. Traditionally, the control of robotic body parts is carried out by feedback control loops. This results, however, in rather machine-like behavior that does not reveal much about what the robot is doing or thinking. (Breeman, 2004)

\subsection{ROBOT EXPRESSIONS BY VOICE AND SOUND}

A robotic sound cliché may be so familiar in our minds, what makes a robotic sound so robotic? Regular pitch and unchanging speed of talking can be two important causes that reflect no excitement or feeling in voice.

Speech Synthesis field can be used to convert text to speech, as in WALL-E (2008) AUTO's voice was produced by a text-to-speech program called MacInTalk (IMDB) [9]. The other step would be utilizing Natural Language Processing field's achievements to give different pitch and speed to same sentences in different situations. Besides scientific studies and efforts in producing voices for robots that can show the emotions, adding some features to a special robot, such as pronouncing a special expression more frequent or with a unique attracting pitch can make it cute and more believable.

In WALL-E (2008), the cockroach has a sound heard when moving, although in real life that sound may not be heard, this can give the idea of adding some unnecessary sound to some robot's actions to form sort of characteristics to them, and this also can help the visitors to become familiar with that sound, let them guess in later times what the robot is doing or going to do, letting them feel that they know the robot.

\section{CHARACTERISTICS}

Can a robot have a photo in a pose that shows the characteristics of it the best? This may be done by set of actions solution that we proposed earlier. In Robots (2005), there is a robot that does many actions while getting angry or when crying he cry in an exaggerated way opening mouth and shaking body, these actions are not necessary to cry or get angry but defines that robot's special character.

B.E.N. a robot in Treasure Planet (2002), that has lost most of his memory in the main part of story, has a special character that talks too much. This can lead to the idea of a making a robot saying some of logical inferences done in its processor loudly, beginning with expressions like, "Well, I think" or "It seems that..."

Saying some random quotations that may even do not match the situation also is another suggestion.

As we mentioned before, Not being perfect makes it more possible to be different among others and that is what exists between human beings and forms the difference, characteristics and identity. Although robots use social learning and but to make them appear different some features can be defined and then decreasing and increasing some features in different robots can give them identity and make them more accepted by people. Although their knowledge are at the same level.

\subsection{AGE}

Age and sex can be defined too. Eva in WALL-E (2008) is female, some shown by the story and some by voice. Besides to voice, color and texture also can help by designing female robots lighter and smoother than male robots. The female robots in 9 (2009) have lighter color with smoother texture.

Making Humanoid Robots More Acceptable Based on the Study of Robot Characters in ... (Fatemeh Maleki) 
Implementing different kind of walking is hard since it is still a challenge to make a robot walk, but adding some visual features or special voice can be used to show age in robots.

\subsection{ACCESSORIES, PETS, INTEREST}

In $W A L L-E$ (2008), when WALL-E, the robot, forgets everything and loses his character he shows not interest to what he was interested in before. Defining some interests for a robot by expressing a sentence or exclamation sound or having it all the time can be a feature to be added to a robot. The robot in Castle in the Sky (1986) who has plants on his shoulder is different and do a different job, although the body shapes are all the same. Number One, a robot in 9 (2009) always have a cane with himself completing his character.

Besides actions and robot body design, accessories or a pet can help a lot in introducing it. In Robots (2005), robots have accessories such as mustache or earrings. The cockroach that always follows $W A L L-E$ robot in $W A L L-E$ (2008) has a role in the robot's identity too. Omnidroid robot in The Incredibles (2004) is more a machine than a robot but have ancestors that can propose the idea of family of robots with differences in sizes or cloths and accessories.

\section{CONCLUSIONS AND FUTURE WORK}

Based on the study of robot characters in animation we extracted features to to be implemented in robots make them are more acceptable. Features in appearance, actions, and accessories can give characteristics and identity to robots but with less effort to resembling precisely to human beings. Animations chosen in this study were also made for adults. Future studies with concentration on the robots that are designed to interact with children can be suggested, this needs a separate study since children conceptions are different and they diagnose a moving object as robot, animal or human in a different way.

\section{ACKNOWLEDGEMENTS}

We would like to thank Dr. Mohammad Reza Hosnaee for his encouragement, Farrokh Yekdaneh whose interest in combining robotics and animation subjects encouraged us and Dr. Fatemeh Hosseini Shakib for her sincere devote to promote animation studies in Art University of Tehran and for her help on Uncanny Valley subject. With special thanks to Disney research team who worked on the robot able to play catch and juggling.

\section{REFERENCES}

[1] Kober, Jens; Glisson, Matthew; Mistry ,Micheal. Playing Catch and Juggling with a Humanoid Robot, Disney Research, 2012. [Online]. Available at http://www.disneyresearch.com/project/juggling_robot/ [Accessed 13 January 2013]

[2] Russell, Stuart; Norvig, Peter. Artificial Intelligence: A Modern Approach,3rd Edition, 2009

[3] Beyond.com, NAO Robots Reach and Teach Autistic Children, http://www.beyond.com/articles/nao-robots-reachand-teach-autistic-children-11957-article.html, last access 3/27/2014

[4] Mori, M. (1970/2012). The uncanny valley (K. F. MacDorman \& N. Kageki, Trans.). IEEE Robotics \& Automation Magazine, 19(2), 98-100. doi:10.1109/MRA.2012.2192811

[5] Nakanoa, Tamami; Katoc, Makoto; Moritoc, Yusuke; Itoid ,Seishi; Kitazawaa ,Shigeru. Blink-related momentary activation of the default mode network while viewing videos, Proceedings of the National Academy of Sciences, December 24, 2012

[6] Dirk Neumann, et. al ,Looking you in the mouth: abnormal gaze in autism resulting from impaired top-down modulation of visual attention, Social Cognitive \& Affective Neuroscience, Volume 1, Issue 3, Pp. 194-202, 2006

[7] van Breemen, A.J.N. Bringing Robots To Life: Applying Principles of Animation To Robots, CHI2004, Vienna, 2004.

[8] Disney Research. http://www.disneyresearch.com/project/juggling_robot/ [Accessed 13 January 2013]

[9] IMDB, MacInTalk, [Online].Available at http://www.imdb.com/name/nm3050831/ [Accessed 13 January 2013] 